\title{
Risk to the Health Care Workers in Present Pandemic of COVID-19- Case Report
}

\author{
Nayab Mustansar* and Rizwan Rafi \\ Department of Nuclear Medicine, Consultant Nuclear Physician, Pakistan
}

*Corresponding author: Nayab Mustansar, Department of Nuclear Medicine, Consultant Nuclear Physician, Islamabad, Pakistan

\begin{tabular}{l} 
ARTICLE INFO \\
\hline Received: 慧 May 01, 2020 \\
Published: 輩 May 15, 2020
\end{tabular}

Citation: Nayab M, Rizwan R. Risk to the Health Care Workers in Present Pandemic of COVID-19- Case Report. Biomed J Sci \& Tech Res 27(4)-2020. BJSTR. MS.ID.004539.

\section{Abstract}

Background: Presently little is known about the effectiveness of personal protective equipment for health care workers who are directly in contact with the patients infected with Novel (SARS- CoV-2) [1,2].

Objective: To assess the clinical picture and risk of the health care worker who come in contact with the COVID 19 patients directly before they were known to be positive patients.

Keywords: COVID19; PCR; Nasopharyngeal Swab; Health Care Workers

\section{Case Report}

The patient was a 56 years old middle-aged lady with diabetes mellitus and hypertension who was hospitalized in January 2020 for community-acquired pneumonia. She had not travel recent travel history to China nor had had contact with anyone known to have COVID-19. She required supplemental oxygen on admission; the following day, she developed respiratory distress that required endotracheal intubation by the emergency airway team and mechanical ventilation in the Intensive Care Unit (ICU). She was then shifted to the ICU (Intensive Care Unit) for intubation. She improved clinically after 5 days of mechanical ventilation and was subsequently extubated to noninvasive ventilation. On the day that the patient was extubated, a nasopharyngeal swab was sent as part of COVID-19 surveillance, and it was positive for SARS-CoV-2 on Polymerase Chain Reaction (PCR) assay [3]. Two other swabs obtained on subsequent days tested positive for SARS-CoV-2.

On the basis of contact tracing, 38 health care workers (Including Doctors, Nursing staff, Ward boys, Sweeper) were identified as having exposure to aerosol-generating procedures for at least 15 minutes at a distance of less than 2 meters from the patient who happened to be COVID-19 Positive. The aerosol-generating procedures included endotracheal intubation, extubation, noninvasive ventilation, and exposure to aerosols in an open circuit [4]. All 38 health care workers were placed under home isolation for 14 days, with daily monitoring for cough, dyspnea, and myalgia and twice-daily temperature measurements. In addition, they had nasopharyngeal swabs scheduled on the first day of home isolation, which could have been day 1, 2, 4, or 5 after last exposure to patient, and a second swab scheduled on day 14 after their last exposure. The swabs were tested for SARS-CoV-2 by using a PCR assay. None of the exposed health care workers developed symptoms, and all PCR tests were negative.

\section{Discussion}

The primary route for the spread of COVID-19 is thought to be through coughing, sneezing, or breathing due to the aerosolized droplets that are expelled, but there. In the situation we describe, $85 \%$ of health care workers were exposed during an aerosolgenerating procedure while wearing a surgical mask, and the remainder were wearing N95 masks. That none of the health care workers in this situation acquired infection suggests that surgical masks, hand hygiene, and other standard procedures protected them from being infected. Our observation is consistent with previous studies that have been unable to show that N95 masks were superior to surgical masks for preventing influenza infection in health care workers [5]. We emphasize, however, that nearly all experts recommend that health care workers wear an N95 mask or equivalent equipment while performing an aerosol-generating 
procedure. We recognize the limitations of this single case report and acknowledge that additional studies are necessary to determine how best to protect health care workers from becoming infected with SARS-CoV while they are providing care for patients with COVID-19. Moreover, the distancing should be maintained at least 2 meters from the COVID19 patient for decreasing the spread of infection. More isolation centers should be established for keeping the patients under observation with protection to the normal healthy health care workers. Though the use of PPE and N95 masks be encouraged in anyway.

\section{References}

1. Wang D, Hu B, Hu C, Zhu F, Liu X, et al. (2020) Clinical characteristics of 138 hospitalized patients with 2019 novel coronavirus-infected pneumonia in Wuhan, China. JAMA 323(11): 1061-1069.

ISSN: 2574-1241

DOI: $10.26717 /$ BJSTR.2020.27.004539

Nayab Mustansar. Biomed J Sci \& Tech Res

(C) This work is licensed under Creative

Submission Link: https://biomedres.us/submit-manuscript.php
2. Pongpirul WA, Pongpirul K, Ratnarathon AC, Prasithsirikul W (2020) Journey of a Thai taxi driver and novel coronavirus. N Engl J Med 382(11):1067-1068.

3. (2020) Ministry of Health Singapore. Ministerial statement on wholeof-government response to the 2019 novel coronavirus (2019-NCOV).

4. (2020) Centers for Disease Control and Prevention. Interim U.S. guidance for risk assessment and public health management of health care personnel with potential exposure in a health care setting to patients with corona disease 2019 (COVID-19).

5. Radonovich LJ, Simberkoff, Bessesen MT, Brown AC, Cummings DAT, et al. (2019) ResPECT investigators. N95 respirators vs medical masks for preventing influenza among health care personnel: a randomized clinical trial. JAMA 322(9): 824-833.

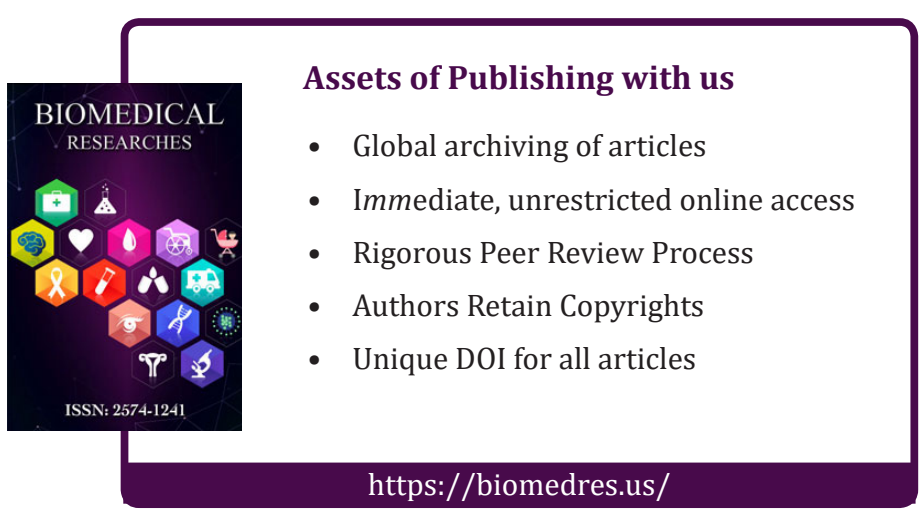

